\title{
Hypertension in Familial Adenomatous
}

\section{Polyposis (FAP) - Think of Cushing's or}

\section{Conn's}

Tom Glass Hopkins M.B.B.S. ${ }^{1}$

Victoria Salem M.R.C.P. ${ }^{1}$

Heba El-Gayar M.R.C.P. ${ }^{1}$

Fausto F Palazzo M.S., F.R.C.S. ${ }^{2}$

Karim Meeran M.D., F.R.C.P., F.R.C.Path ${ }^{1}$

Tricia Tan M.R.C.P. ${ }^{1}$

1. Endocrine Unit, Imperial College Healthcare NHS Trust, Hammersmith Hospital, Du Cane Road, London W12 OHS.

2. Department of Endocrine Surgery, Imperial College Healthcare NHS Trust, Hammersmith Hospital, London W12 OHS. 
A 36-year-old man was found to be hypertensive by his GP: $210 / 110 \mathrm{mmHg}$. He had been diagnosed with familial adenomatous polyposis (FAP) in childhood, and had undergone a total colectomy at the age of 17 . The patient had been under endoscopic surveillance since, with a recent polyp count of 50-60, and remained problem-free. The following month, as part of his routine FAP screening programme, the patient underwent an abdominal CT scan. This revealed bilateral macronodular adrenal hyperplasia. The largest of these nodules measured 4 by $2 \mathrm{~cm}$ on the right and 2.7 by $2.9 \mathrm{~cm}$ on the left (see Figure 1 ).

On assessment in our unit, the patient was found to be hypertensive at $150 / 100$ $\mathrm{mmHg}$ on lisinopril and amlodipine. Examination revealed a well-healed midline laparotomy scar, and no clinical features suggestive of Cushing's syndrome. Serum potassium concentration was normal at $3.8 \mathrm{mmol} / \mathrm{L}$. Other biochemical investigations excluded Cushing's syndrome and phaeochromocytoma.

With confounding antihypertensive medications discontinued, plasma aldosterone measured $590 \mathrm{pmol} / \mathrm{L}$, plasma renin activity (PRA) $0.2 \mathrm{pmol} / \mathrm{ml} \cdot \mathrm{hr}$, giving an aldosterone:PRA ratio of 2950 , where values $>2000$ are considered highly suggestive of primary hyperaldosteronism. ${ }^{1}$ Saline infusion did not suppress aldosterone to $<280 \mathrm{pmol} / \mathrm{L}$, further supporting this diagnosis.

Selective adrenal vein sampling was undertaken in September 2009. The cortisolcorrected aldosterone ratio in the left adrenal vein $\left(A / C_{L}\right)$ was 3.05 versus a ratio of 0.74 in the right adrenal vein $\left(A / C_{R}\right)$, with a ratio of 0.80 in the low inferior vena cava $\left(A / C_{\text {IVC }}\right)$. The dominant to non-dominant ratio $\left(A / C_{L}: A / C_{R}\right)$ of 4.15 was above the 
recommended cut-off of 4.0 for diagnosing unilateral aldosterone hypersecretion with $95 \%$ sensitivity and $100 \%$ specificity. ${ }^{1}$ Furthermore, the non-dominant to peripheral ratio $\left(A / C_{R}: A / C_{I V C}\right)$ of $<1$ confirmed that autonomous secretion of aldosterone was confined to the left.

There were no radiological features of the right adrenal mass suspicious of malignancy apart from its size. With the finding of dominant left-sided aldosterone secretion in this case, it was decided that the patient would benefit from laproscopic left adrenalectomy, and he is currently awaiting surgery.

FAP is associated with numerous extra-intestinal manifestations, with which the attending physician should be familiar. ${ }^{2}$ Adrenal gland enlargement is commoner in patients with FAP above the expected ascertainment bias due to frequent abdominal imaging. In a series of 738 FAP patients, $14(7.4 \%)$ were found to have adrenal enlargement, compared to $0 \cdot 6-3.4 \%$ in the general population. ${ }^{3}$ Of these, two were hypertensive and required surgical removal of cortisol-secreting tumours. The histology revealed adrenal carcinoma in one, and nodular hyperplasia in the other. In another follow-up study of 30 FAP patients with adrenal adenomas, 3 required adrenalectomy: one for a phaeochromocytoma, one for a non-functioning, malignant tumour, and one for an enlarging, non-functioning, benign adenoma. ${ }^{4}$ Thus, the majority of adrenal tumours associated with FAP are non-functioning and require no treatment unless they enlarge. However, it is important to diagnose those rare cases that are associated with hormone hypersecretion, as these are surgically treatable. All FAP patients with incidentally discovered adrenal masses should have regular blood pressure measurements and be screened for possible Cushing's syndrome 
(using a 9 am ACTH level or dexamethasone suppression test), Conn's syndrome (using an aldosterone:PRA ratio), and phaeochromocytoma (with urine catecholamine or metanephrine excretions). ${ }^{4}$

This case report describes aldosterone-secreting adrenal enlargement in a hypertensive patient with FAP. Recently, an association between tumour-suppressor APC (adenomatous polyposis coli) gene mutations and hyperaldosteronism has been proposed. Mice heterozygous for a truncating $A P C$ mutation had higher aldosterone levels than controls, with corresponding increases in urinary potassium excretion, despite the presence of hypertension and plasma volume expansion. This suggests a potential pathophysiological link between the causative $A P C$ mutation and hyperaldosteronism in FAP. ${ }^{5}$

\section{Legends}

Figure 1. Coronal section of unenhanced abdominal CT of a patient with familial adenomatous polyposis (FAP) and noted to be hypertensive one month earlier. Bilateral adrenal enlargement highlighted.

\section{Contributions}

TGH, VS, HEG, FFP, KM and TT: diagnosis and patient management. Written consent to publish was obtained from the patient.

\section{Conflicts of Interests}

None declared. 


\section{References}

1. Funder JW, Carey RM, Fardella C, Gomez-Sanchez CE, Mantero F, Stowasser M, et al. Case detection, diagnosis, and treatment of patients with primary aldosteronism: an Endocrine Society clinical practice guideline. J Clin Endocrinol Metab. 2008;93:3266-81.

2. Groen EJ, Roos A, Muntinghe FL, Roelien EH, de Vries J, Kleibeuker KH, et al. Extra-intestinal manifestations of familial adenomatous polyposis. Ann Surg Oncol 2008;15:2439-50.

3. Marchesa P, Fazio VW, Church JM, McGannon E. Adrenal masses in patients with familial adenomatous polyposis. Dis Colon Rectum. 1997;40:1023-8.

4. Will OC, Hansmann A, Phillips RK, Palazzo FF, Meeran K, Marshall M, et al. Adrenal incidentaloma in familial adenomatous polyposis: a long-term follow-up study and schema for management. Dis Colon Rectum. 2009;52:1637-44.

5. Bhandaru M, Kempe DS, Rotte A, Rexhepaj R, Kuhl D, Lang F. Hyperaldosteronism, hypervolemia, and increased blood pressure in mice expressing defective APC. Am J Physiol Regul Integr Comp Physiol. 2009;297:R571-5. 